\title{
Microecological treatment of hyperuricemia using Lactobacillus from pickles
}

\author{
Yuanxun Xiao ${ }^{\dagger}$, Congxin Zhang ${ }^{*}$, Xianli Zeng ${ }^{\dagger}$ and Zhichao Yuan ${ }^{\dagger}$
}

\begin{abstract}
Background: Hyperuricemia is one of the important risk factors for gout, arteriosclerosis, cardiovascular and cerebrovascular disease. Lactobacillus has attracted much attention due to its role in the regulation of intestinal function and tumor resistance, but its ability to reduce uric acid is unclear. Pickles are a traditional fermented food rich in lactic acid bacteria (LAB).

Results: $L A B$ strains were isolated from 18 pickles and their tolerance to acid bile salts, trypsin, pepsin were evaluated after screening by nucleoside degradation. $16 \mathrm{~S}$ rDNA sequence analysis was used to identify LAB strains. Furthermore, we established rat model of hyperuricemia and demonstrated that Lactobacillus could alleviate hyperuricemia and reduce kidney injury.

Conclusion: This study suggests that microecological treatment with Lactobacillus represents a feasible option for patients with chronic hyperuricemia.
\end{abstract}

Keywords: Lactic acid bacteria, Pickles, Hyperuricemia, Microecology, Uric acid

\section{Background}

Hyperuricemia is a progressive chronic disease without obvious clinical symptoms [1]. Abnormal uric acid in the blood can induce gout, and is an important risk factor for arteriosclerosis, cardiovascular and cerebrovascular disease [2-4]. It is known that Western diet is rich in purine and adenine. In addition, the purine compounds disodium 59-guanylate and disodium 59-inosinate are the main components of many flavor enhancers that are widely used in modern food production [5]. At present, several clinically available drugs can control acute gout, but most patients with hyperuricemia are unable to achieve long-term control due to high purine diets and poor treatment efficacy [6]. For example, allopurinol can induce hypersensitivity syndrome, which may lead to the death of patients [7]. Therefore, the use of purine

\footnotetext{
* Correspondence: zhangd616@gmail.com

${ }^{\dagger}$ Yuanxun Xiao, Xianli Zeng and Zhichao Yuan contributed equally to this work.

Department of LiWan Hospital, Third Affiliated Hospital of Guangzhou Medical University, Guangzhou 510150, Guangdong, China
}

compound degrading probiotics is a promising alternative for the prevention of hyperuricemia.

Lactic acid bacteria (LAB) are Gram positive, acidtolerant, fermenting rods or cocci that produce lactic acid as the major metabolic end-product of carbohydrate fermentation [8]. In recent years, Lactobacillus has gained interest due to the ability to regulate molecular microbial ecology in the human body [9]. Some LAB strain could reduce serum uric acid level by assimilating purine nucleosides because it produced inosine hydrolase which degraded inosine in vitro, and reversed highfructose induced increase of uric acid by reducing inosine [10-12].

Pickles are traditional fermented food that produce abundant LAB in a short timeframe under sealed air and acidic conditions. However, whether LAB from traditional pickles can reduce uric acid levels remains unclear. In China, it is still not widely accepted to take daily supplement of probiotics such as LAB, while most Chinese eat pickles due to the low price and easy accessibility. Therefore, in this study we aimed to investigate 
the potential of Lactobacillus from pickles as a new microecological approach for the clinical treatment of hyperuricemia. We established rat model of hyperuricemia and examined whether Lactobacillus could alleviate hyperuricemia and reduce kidney injury in the rats.

\section{Results}

\section{Isolation of $L A B$ strains}

LAB strains were isolated from most pickles, but large differences in the colonies across the pickles were observed (Table 1). Fourteen strains from 18 different pickles were screened as gram staining positive and $\mathrm{H}_{2} \mathrm{O}_{2}$ test negative (Table 2). LAB were predominantly rod shaped and globular (Fig. 1b-e). We also observed colonies containing various LAB types (Fig. 1b-e).

\section{Inosine and guanosine degradation by LAB strains}

HPLC chromatograms showed that peak areas of inosine and guanosine were 17,547.2 and 17,522.1, respectively (Fig. 2a, b). Due to favorable discrimination, we were able to distinguish inosine and guanosine (Fig. 2c). We identified 14 strains with various ability to degrade nucleosides. Among the strains, S1, S5, S12 and S14 degraded more than $40 \%$ of nucleosides, and were thus selected for further analysis (Fig. 2d).

\section{Characterization of candidate strains}

S1, S5, S12, S14 strains had improved tolerance to acid, pepsin, bile salts and trypsin as the OD values for these strains increased under the treatment conditions. In particular, S12 strain had the highest tolerance among the four strains (Fig. 3a-d). Notably, we found irregularity in OD in control group although we repeated experiments, and because we used liquid MRS medium as control, the pattern of OD change may be due to the reaction of liquid MRS with trypsin over time.

\section{Identification of candidate strains}

Four candidate strains possessed different types of LAB. More than $75 \%$ of the four strains were Lactobacillus paracasei among which S12 strain had a content as high as $99 \%$. Other LAB present included Lactobacillus plantarum, Lactobacillus brevis and Pantoea agglomerans (Fig. 4a, b). Compared to the Silva database (v123), the four candidate strains were the same type of Lactobacillus paracasei (over 97\% homology. (Fig. 4c). We selected S12 strain for further analysis because of the similarity. We also found that pickles included other strains in the different Heatmap level. Firmicutes, Bacteroidetes and Proteobacteria were shown in the phylum levels, and the Firmicutes were over 95\% (Fig. 5a). The heatmap showed that several microbial colonies coexisted, but Lactobacillus was dominant bacterial community (Fig. 5b-f).

\section{S12 strain alleviates hyperuricemia in rats}

Upon kidney assessment, we found that the kidneys of S12 group were only partially damaged compared to

Table 1 The number of $L A B$ colonies isolated from pickles

\begin{tabular}{|c|c|c|c|c|}
\hline Number & Product & Origin & Total bacterial colony ${ }^{a}$ & Lactobacillus colony $^{b}$ \\
\hline 1 & Lactobacillus Yogury & GuangZhou, GuangDong & 2 & 1 \\
\hline 2 & Erie milk & HuErHaoTe, Inner Mongolia & 1 & 1 \\
\hline 3 & Lllie Lactobacillus & HuErHaoTe, Inner Mongolia & 18 & 1 \\
\hline 4 & Ferment Powder (lacto) & HaiDian, BeiJing & 1 & 1 \\
\hline 5 & Stewed Bamboos & YongFeng, JiangXi & 6 & 0 \\
\hline 6 & Sour Beans & ChengDu,SiChuan & 25 & 0 \\
\hline 7 & Kraut & MeiShan, SiChuan & 18 & 2 \\
\hline 8 & Pickle (vegetables) & ChengDu,SiChuan & 65 & 1 \\
\hline 9 & Pickle (vegetables) & BaoShan, YunNan & 59 & 1 \\
\hline 10 & Fish Sauerkrant & WeiYan,SiChuan & 9 & 1 \\
\hline 11 & Pickle (lotus root) & JinZhou, HuBei & 24 & 1 \\
\hline 12 & Pickle (cabbage) & XinMin,ShenYang & 80 & 1 \\
\hline 13 & Pickle (vegetables) & XinDu, ChengDu & 7 & 1 \\
\hline 14 & Pickle (vegetables) & ShenYang,LiaoNing & 22 & 1 \\
\hline 15 & Pickle (vegetables) & QingZhen, GuiZhou & 36 & 1 \\
\hline 16 & Pickle (lettuce) & YueYang, HuNan & 71 & 0 \\
\hline 17 & Pickle (kelp) & YueYang, HuNan & 32 & 0 \\
\hline 18 & Pickle (cabbage) & ChengDu, SiChuan & 6 & 1 \\
\hline
\end{tabular}

Note: a. Total number of bacterial colony grown on MRS medium. b. Total number of LAB colony grown on MRS medium 
Table 2 The characteristics of different LAB strains

\begin{tabular}{|c|c|c|c|c|c|}
\hline Strain & Source & Form & $24 \mathrm{~h} \mathrm{pH}^{\mathrm{a}}$ & $\mathrm{H}_{2} \mathrm{O}_{2}$ test $^{\mathrm{b}}$ & 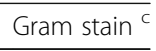 \\
\hline S1 & Lactobacillus Yogury & Rhabditi form & $6.31-3.86$ & - & + \\
\hline S2 & Erie milk & Rhabditi form & $6.33-3.89$ & - & + \\
\hline S3 & Lllie Lactobacillus & Rhabditi form & $6.33-3.84$ & - & + \\
\hline S4 & Kraut & Rha/Sph & $6.30-3.93$ & - & + \\
\hline S5 & Kraut & Spherality & $6.32-3.75$ & - & + \\
\hline S6 & Pickle (vegetables) & Rha/Sph & $6.32-3.85$ & - & + \\
\hline S7 & Pickle (vegetables) & Rhabditi form & $6.29-3.81$ & - & + \\
\hline S8 & Fish Sauerkrant & Rhabditi form & $6.31-3.91$ & - & + \\
\hline S9 & Pickle (lotus root) & Rhabditi form & $6.32-3.84$ & - & + \\
\hline S10 & Pickle (cabbage) & Rhabditi form & $6.31-3.83$ & - & + \\
\hline S11 & Pickle (vegetables) & Rhabditi form & $6.32-3.84$ & - & + \\
\hline S12 & Pickle (vegetables) & Rhabditi form & $6.31-3.80$ & - & + \\
\hline S13 & Pickle (vegetables) & Rhabditi form & $6.31-3.81$ & - & + \\
\hline S14 & Pickle (cabbage) & Rhabditi form & $6.29-3.79$ & - & + \\
\hline
\end{tabular}

Note: a. $24 \mathrm{~h}$ change of pH. b. $\mathrm{H} 2 \mathrm{O} 2$ test determined the presence of catalase, an enzyme that degrades $\mathrm{H} 2 \mathrm{O} 2$. Negative (-) indicated the absence of catalase. $\mathrm{c}$. Gram staining positive $(+)$ indicated the bacteria had thick cell wall. Most LAB could produce acid, lack catalase and are Gram ${ }^{+}$

model group. Renal tubular epithelial cells displayed edema and degeneration in S12 group, but renal glomerulus and tubule structures were still visible. In contrast, model group displayed significant renal damage, including edema and degeneration of the edulla and cortex, and capillary congestion (Fig. 6a-c). Serum UA and BUN of each model group significantly differed from control group on day $10(P<0.05)$. Thus hyperuricemia model was successfully established under our conditions. On day 20, serum UA levels in S12 group were
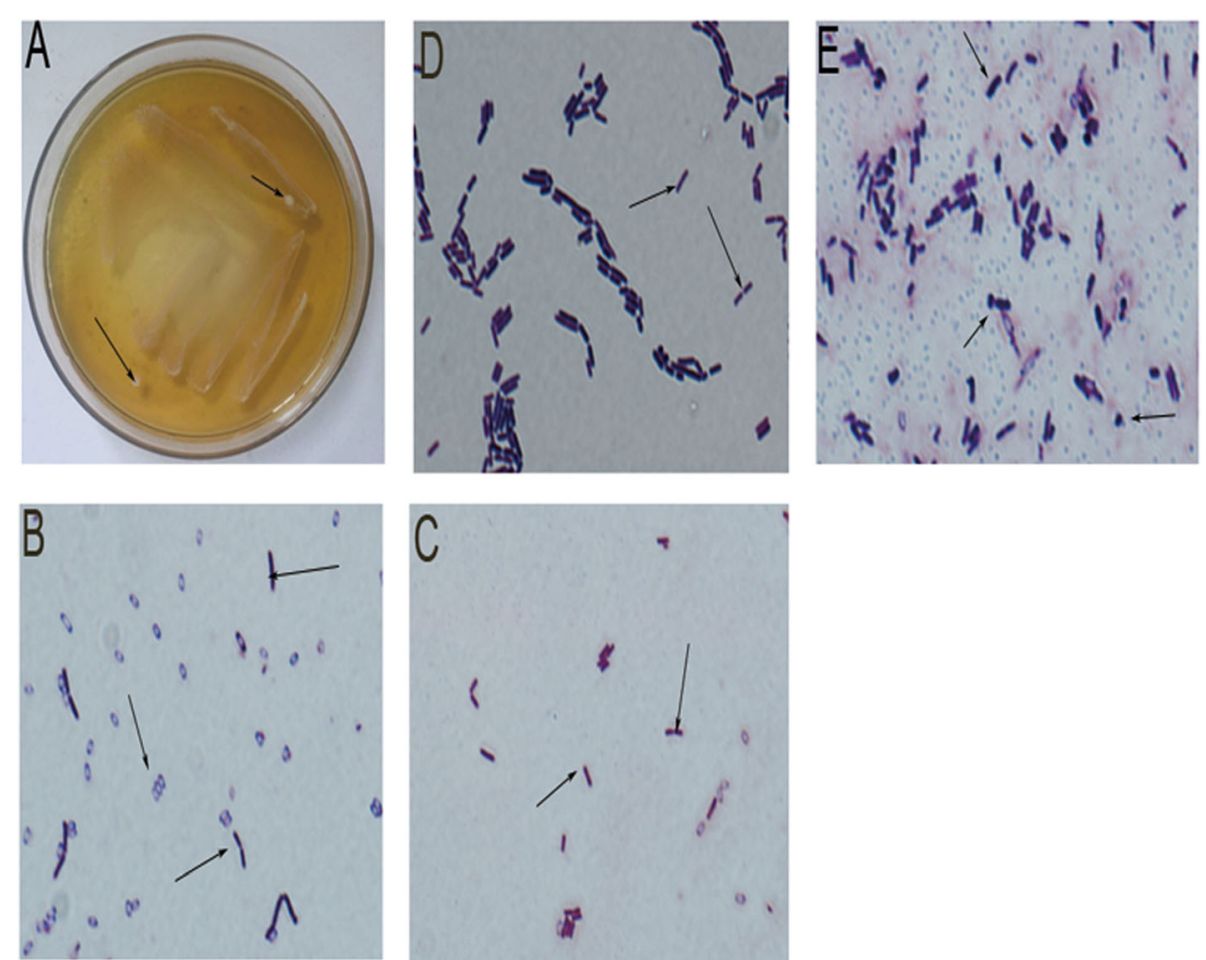

Fig. 1 Isolation of LAB strains. a. MRS culture plate. b. S1 strains showed Rhabditi form under high-power microscope (100x oil). c. S5 strains showed spherality form under high-power microscope (100x oil). d. S12 strains showed Rhabditi form under high-power microscope (100x oil). e. S14 strains showed Rhabditi form observed under high-power microscope (100x oil) 
A

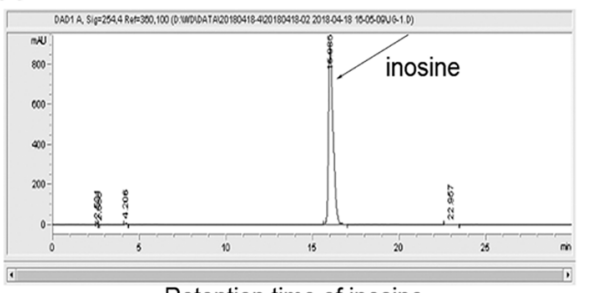

B

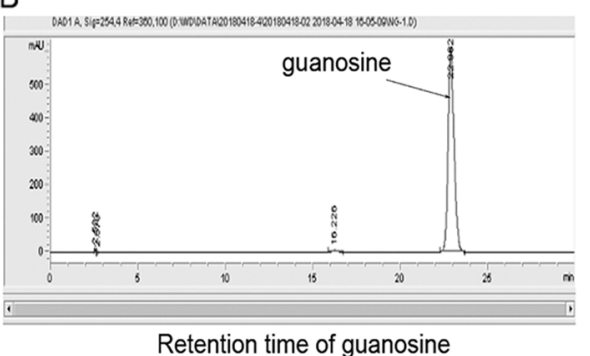

C

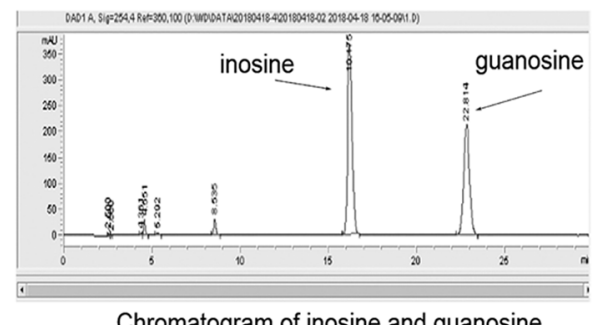

Chromatogram of inosine and guanosine
$\mathrm{D}$
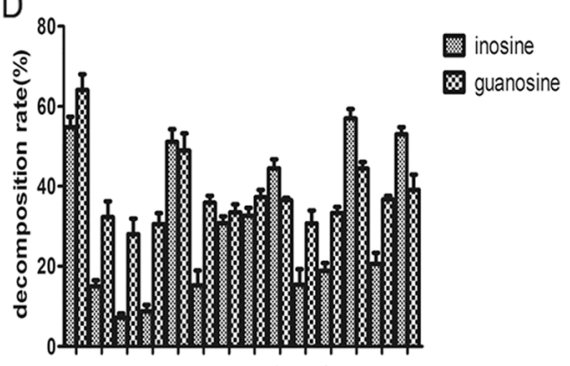

$s^{1} s^{2} s^{3} s^{x} s^{6} s^{6} s^{1} s^{8} s^{9} s^{0} s^{1} s^{2} s^{2} s^{3} s^{x}$

Fig. 2 The degradation of inosine and guanosine by LAB strains. a. The retention time of inosine in HPLC chromatogram. $\mathbf{b}$. The retention time of guanosine in HPLC chromatogram. $\mathbf{c}$. The retention time of inosine and guanosine in HPLC chromatogram. $\mathbf{d}$. The assimilating speeds of inosine and guanosine showed variations in LAB S1-S14 strains

lower compared to model group $(P<0.05)$, but serum $\mathrm{Cr}$ and $\mathrm{BUN}$ increased in all groups compared to control group $(P<0.05)$. There were no changes in serum $\mathrm{Cr}$ or BUN levels in control group at the various time points (Fig. 6d-f).

\section{Discussion}

Purine rich foods such as meat and seafood as well as alcoholic beverages potently exacerbate hyperuricemia. Probiotics with the ability to degrade purine compounds in food have been investigated for the prevention of hyperuricemia [10-13]. Pickles are typical representative of Chinese traditional fermented food as a rich natural source of LAB [14]. Microorganisms degrade nucleosides mainly through the biosynthesis of nucleoside hydrolases. Nucleoside hydrolases break the glycoside bonds of nucleosides and release nitrogenous bases, which are further processed by xanthine dehydrogenase to produce uric acid [15, 16]. Thus the existence of nucleoside hydrolase in the strain can be proven by the degradation of nucleoside substances such as guanosine and inosine.

To determine whether the tested strains can survive in the gastrointestinal environment and colonize successfully, the biological characteristics of the four candidate strains were evaluated. The gastrointestinal tract is rich in digestive enzymes, with strong acidic condition to inhibit bacterial growth [11]. Generally, it takes $4 \mathrm{~h}$ for food to enter the intestinal tract from the mouth for digestion and absorption. All four strains were well tolerated in the simulated GI environment [11]. We found Lactobacillus paracasei to be the representative strain isolated from traditional pickles by $16 \mathrm{~S}$ rDNA sequence analysis [17]. S12 strain had the highest similarity of Lactobacillus paracasei (99.33\%), and was used for in vivo animal experiments. In addition, we investigated the taxonomy of microbial colonies in pickles, including Firmicutes, Bacteroidetes and Proteobacteria. These data help better understanding of the microbial environment of pickles.

We established a hyperuricemia model in rat by combining yeast-extract with oxonic acid potassium [18]. However, it has been difficult to establish animal hyperuricemia model because commonly used laboratory animals such as rats express urate oxidase, and kidney damage can occur over time [19]. On day 20, UA levels of S12 treatment group were significantly lower than 


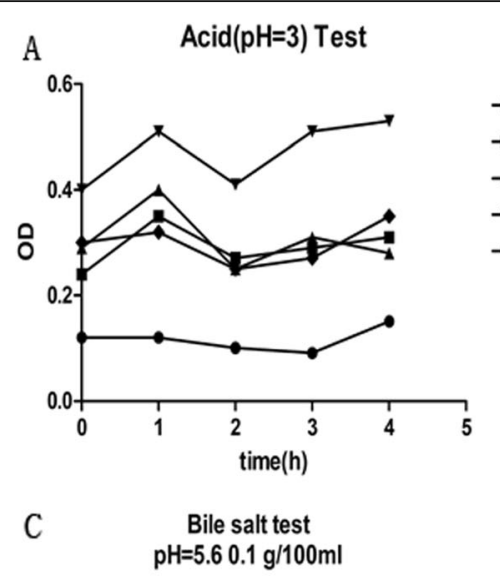

B Gastric protease resistance test $\mathrm{pH}=30.3 \mathrm{~g} / 100 \mathrm{ml}$
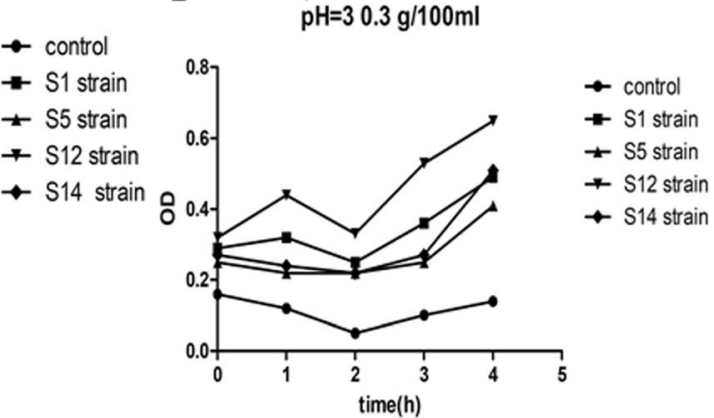
D Trypsin resistance test
$\mathrm{pH}=80.1 \mathrm{~g} / 100 \mathrm{ml}$
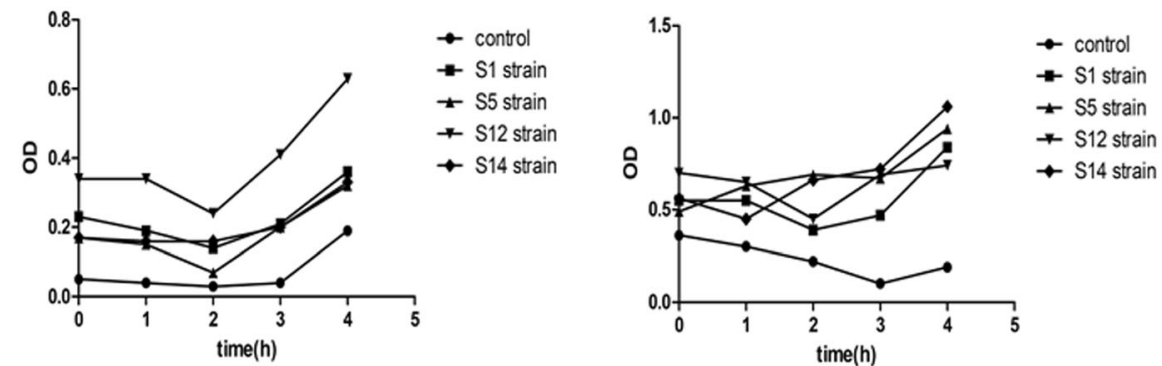

Fig. 3 The growth of strains in different conditions. Different strains (S1, S5, S12 and S14) were cultured in liquid MRS medium in the presence of different substances, and OD values were measured over time. $\mathbf{a}$. The growth of strains in acid-containing medium. $\mathbf{b}$. The growth of strains in gastric protease-containing medium. $\mathbf{c}$. The growth of strains in gastric bile-containing medium. $\mathbf{d}$. The growth of strains in gastric trypsincontaining medium. Control: liquid MRS only

model group, but BUN and Cr of S12 treatment group and model group were higher than control group. Thus Lactobacillus can reduce UA to some extent. These results are consistent with previous reports $[20,21]$. The reason is that lactobacillus can hydrolyze nucleosides in food and compete with the intestinal epithelium to absorb nucleosides, but does not influence the free nucleosides in food [22]. On day 20, although there was no significant difference in $\mathrm{Cr}$ and BUN between S12 treatment groups and model group, model group tended to worsen kidney damage. However, we observed no such toxicity for Lactobacillus, suggesting that it provides protection against acute hyperuricemia induced kidney injury [23, 24]. Considering the short duration of this study, long-term observation of probiotic therapy is still needed. Currently, only two studies have reported the effects of probiotics to reduce hyperuricemia in rat model $[11,12]$. Our results are consistent with these studies. However, our study developed better hyperuricemia rat model because the rats were administrated with yeastextract containing high contents of purine and oxonic acid potassium simultaneously to induce hyperuricemia, while in the other two studies the animals were either administrated with oxonic acid potassium alone or administrated with high purine die and oxonic acid potassium consecutively $[11,12]$. Although our experimental setup has limitation because we did not employ known effective drug for hyperuricemia as positive control for LAB, our data based on hyperuricemia model may better reveal beneficial effects of LAB for hyperuricemia therapy.

\section{Conclusion}

In summary, we successfully screened Lactobacillus paracasei from 18 traditional pickles and demonstrated its ability to degrade nucleosides in vitro and UA in vivo. Rat model of chronic hyperuricemia revealed the potential of Lactobacillus to reduce kidney damage. These results suggest that microecological treatment with Lactobacillus represents a feasible option for patients with chronic hyperuricemia, especially those with impaired renal function.

\section{Methods}

\section{Isolation of LAB}

Eighteen samples of pickles (pickled Chinese cabbage) were purchased from different parts of China. Each sample $(1 \mathrm{~g})$ was blended with $9 \mathrm{~mL}$ of pure water and diluted sample was plated onto MRS medium (Difco, USA), and incubated at $37^{\circ} \mathrm{C}$ until single colonies grew. 


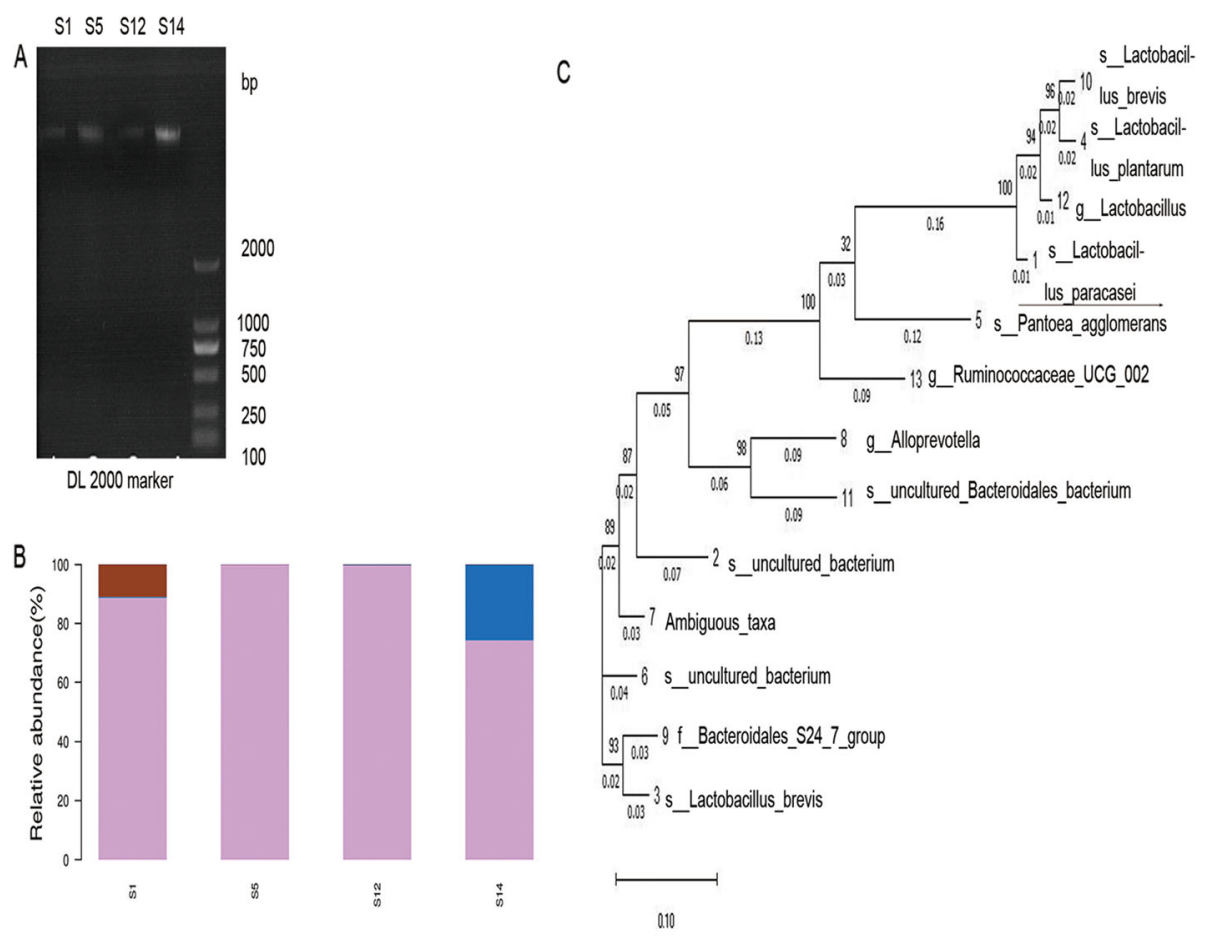

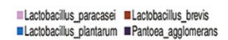

Fig. 4 Phylogenetic tree of S1, S5, S12 and S14 strains based on $16 \mathrm{~S}$ rDNA sequences. a. PCR results. $\mathbf{b}$. The ratio of each strain in the samples. c. The phylogenetic tree based on the $16 \mathrm{~S}$ rDNA sequences. Reference sequences were obtained from the GenBank nucleotide sequence database (NCBI), including KR066436.1, EF097420.1.1396, DQ824024.1, KX519704.1, GQ853416.1, DQ014606.1, EU791061.1, EF096774.1, AB934477.1, GU295951.1, AB702765.1, DI343235.1, DQ808601.1

Each single colony was preliminarily screened for LAB by gram staining, $\mathrm{H}_{2} \mathrm{O}_{2}$ test and microscopic evaluation $(100 \times$ oil $)$. All samples were stored at $-80^{\circ} \mathrm{C}$.

\section{Measurement of inosine and guanosine levels}

HPLC was used to detect inosine and guanosine levels simultaneously. $33.7 \mathrm{mg}$ inosine and $35.7 \mathrm{mg}$ guanosine were dissolved in $100 \mathrm{~mL} \mathrm{~K}{ }_{3} \mathrm{PO}_{4}$ solution $(100 \mathrm{mmol} / \mathrm{L}$, $\mathrm{pH}$ 7.0) and $20 \mathrm{uL}$ of each solution was injected into a HPLC device (LC-20A, Shinadzu Corporation, China) equipped with a column 5C18-AR-II $(4.6 \times 250 \mathrm{~mm}$, Cosmosil, China). The final flow phase $(0.1 \mathrm{umol} / \mathrm{L}$ $\mathrm{NaHPO}_{4}, 0.187 \mathrm{~mol} / \mathrm{L} \mathrm{H}_{3} \mathrm{PO}_{4}$ ) was performed at a flow rate of $1 \mathrm{~mL} / \mathrm{min}$, a wavelength of $254 \mathrm{~nm}$, a retention time of 40 mins and a column temperature of $30^{\circ} \mathrm{C}$.

To assess their ability to degrade inosine and guanosine, $1 \%$ LAB was inoculated in $10 \mathrm{~mL}$ of liquid MRS and cultured for $48 \mathrm{~h}$ at $37^{\circ} \mathrm{C}$ in oxygen free conditions. MRS solution $(2 \mathrm{~mL})$ was then centrifuged at $4000 \times g$ for 5 mins at $4{ }^{\circ} \mathrm{C}$. The sediment was rinsed with pure water and resuspended in $750 \mathrm{uL}$ of inosine-guanosine solution at $37^{\circ} \mathrm{C}$ for $60 \mathrm{~min}$ with shaking $(120 \mathrm{rpm})$. The solutions were analyzed by HPLC as described above. The rate of inosine and guanosine degradation by LAB strains was assessed according to the following equation: $(v=[(0.9 C-X) 0.9 C] \times 100 \%, X$ : the remaining area of ionisine and guanosine on the chromatogram $\mathrm{V}=$ speed of degradation $(\mathrm{g} / \mathrm{L} / \mathrm{min})$.

\section{Acid tolerance assay}

Candidate $\mathrm{LAB}$ strains (1\%) were inoculated in liquid MRS (Difco, USA), and cultured for $24 \mathrm{~h}$ at $37^{\circ} \mathrm{C}$ under oxygen free conditions in anaerobic chest with AnaeroPack (Beijing BY Tech, China). Liquid MRS (5\%) was then injected into $10 \mathrm{ml}$ MRS and cultured at $37^{\circ} \mathrm{C}$. ODs were assessed at hourly intervals from 0 to $4 \mathrm{~h}$ using a spectrophotometer (Nanodrop, Gene Limited, China). Briefly, the number of bacteria in the bacterial suspension per unit volume was measured at OD600. A standard curve was established with the corresponding OD value and bacteria number (OD600 $=1,2 \times 10^{9} \mathrm{cfu} /$ $\mathrm{ml})$.

\section{Bile tolerance assay}

Candidate LAB strains (1\%) were inoculated in liquid MRS and cultured for $24 \mathrm{~h}$ at $37^{\circ} \mathrm{C}$ under oxygen free conditions. Liquid MRS (5\%) was then injected into 10 $\mathrm{ml}$ of MRS (pH 5.6, $0.1 \mathrm{~g} / 100 \mathrm{~mL}$ ) and cultured at $37^{\circ} \mathrm{C}$. 


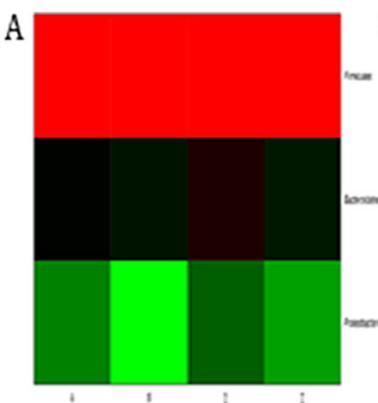

Phylum

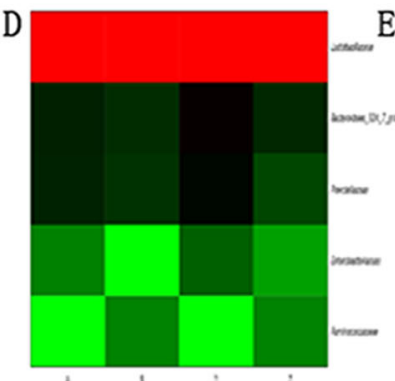

Family

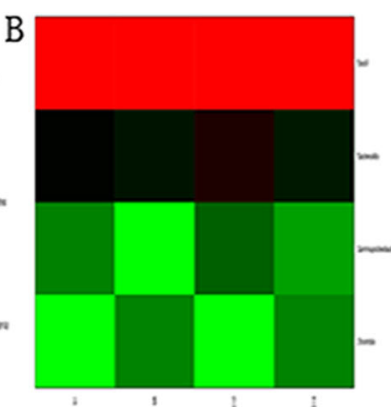

Class

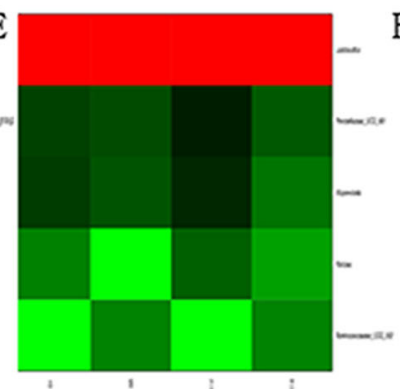

Genus

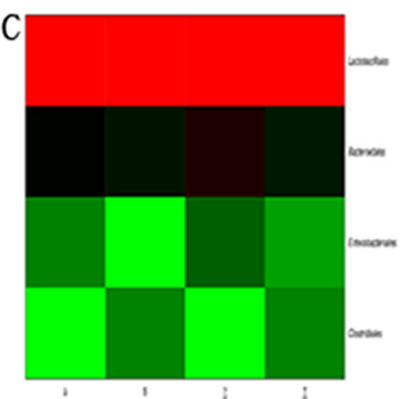

Order

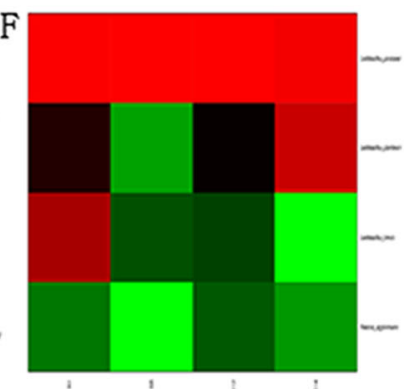

Species

Fig. 5 Different abundance of colonies in the Heatmap. a. Top 30 of the sample abundance in phylum level. b. Top 30 of the sample abundance in class level. c. Top 30 of the sample abundance in order level. $\mathbf{d}$. Top 30 of the sample abundance in family level. e. Top 30 of the sample abundance in genus level. f. Top 30 of the sample abundance in species level

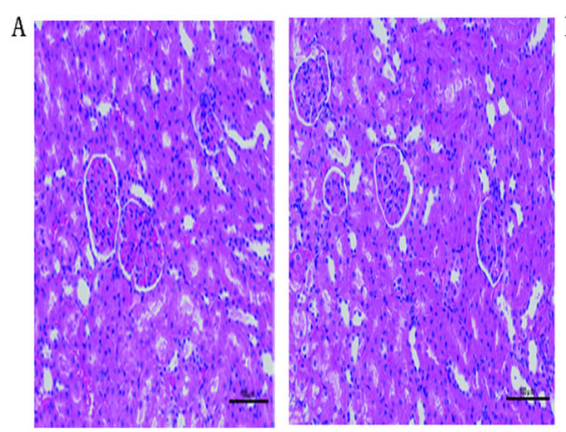

Control group (HEx200)

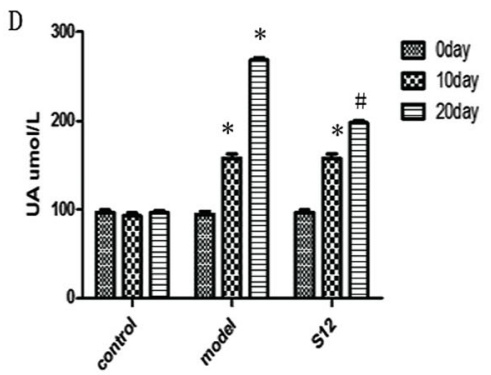

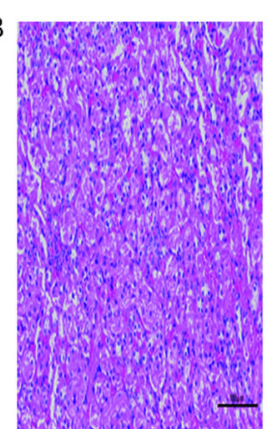

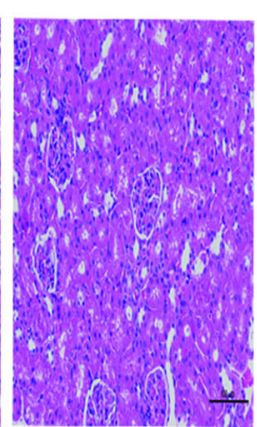

Model group $(20 \mathrm{~g} / \mathrm{kg}+350 \mathrm{~m} / 100 \mathrm{~g})(\mathrm{HEx} 200)$

E

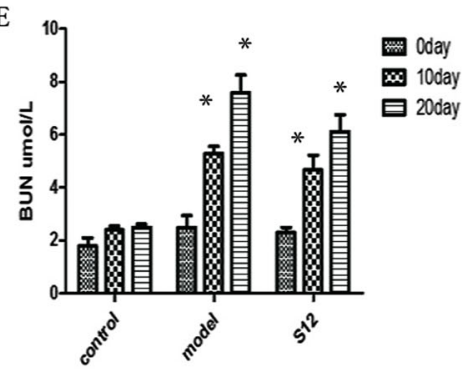

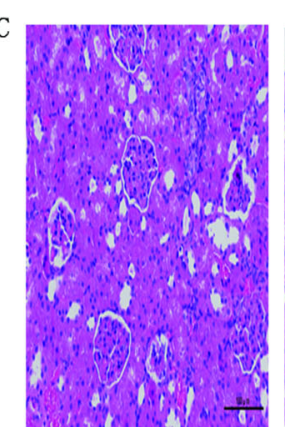

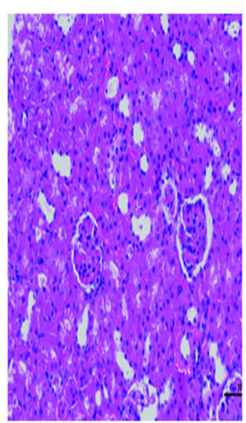

S12 group $(1.5 \times 109 \mathrm{cfu} / \mathrm{m} 1)(\mathrm{HEx} 200)$

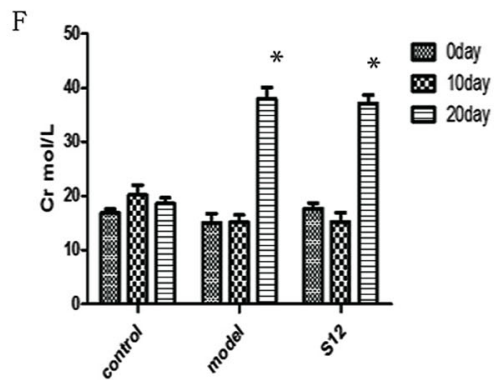

Fig. 6 S12 strain alleviates hyperuricemia in rats. a, b, c. Histological analysis of kidney tissue sections on day 20 in different rat groups. d. The levels of serum UA in different rat groups $(n=5)$. ${ }^{*} P<0.05$ compared with control group. \#P<0.05 compared with model group. e. The levels of serum BUN in different rat groups $(n=5)$. ${ }^{*} P<0.05$ compared with control group. $\mathbf{f}$. The levels of serum $C r$ in different rat groups $(n=5)$. ${ }^{*}<0.05$ compared with control group 
ODs were assessed at hourly intervals from 0 to $4 \mathrm{~h}$ using a spectrophotometer.

\section{Pepsin and trypsin tolerance assay}

Candidate $\mathrm{LAB}$ strains (1\%) were inoculated in liquid MRS and cultured for $24 \mathrm{~h}$ at $37^{\circ} \mathrm{C}$ under oxygen free conditions. Liquid MRS (5\%) was injected into $10 \mathrm{ml}$ MRS of $\mathrm{pH} 3$, pepsin $(0.3 \mathrm{~g} / 100 \mathrm{~mL})$ and of $\mathrm{pH} 8$, trypsin $(0.1 \mathrm{~g} / 100 \mathrm{~mL})$, respectively, and cultured at $37^{\circ} \mathrm{C}$. ODs were assessed at hourly intervals from 0 to $4 \mathrm{~h}$.

\section{Sequencing}

$16 \mathrm{~S}$ rDNA sequencing was performed following previously described method [19]. Briefly, DNA was extracted using the CTAB method and DNA concentrations were quantified using a NanoDrop and agarose gel electrophoresis. Illumina Miseq sequencing was used to perform multiple splicing. VSEARCH (v2.4.2) software was used to classify all high-quality sequences with $97 \%$ similarity for OTU classification and beta diversity analysis. Finally, an evolutionary tree was built and compared to the Silva database (v123). The microbial communities of the four samples were compared and analyzed according to OTU classification. Since there were multiple OTUs corresponding to the same genus or species, Heatmap was drawn and the TOP30 species in richness ranking were provided.

\section{Animal model}

Animal experiments were carried out at Guangzhou Medical University (Guangzhou, China) following NIH guidelines for the Care and Use of Laboratory Animal and the protocols were approved by Animal Use and Care of Committee of Guangzhou Medical University (Approval No. GMU00327). Male SD rats (42 days old) were provided by Animal Center of the Traditional Chinese Medicine University of Guangzhou, China (SCXK 2013-0034). The rats were housed in a specific pathogen-free (SPF) laboratory at a constant temperature $\left(22 \pm 1{ }^{\circ} \mathrm{C}\right)$ and $40-50 \%$ humidity, with a 12 $\mathrm{h}$ light/dark cycle and free access to chow and water. The rats were randomly divided into three groups $(n=$ 10): (1) control; (2) hyperuricemia model; (3) S12 strain treatment. All groups (excluding controls) were administered with yeast extract $(20 \mathrm{~g} / \mathrm{kg}$ body weight) by gavage combined with oxygen oxazine acid potassium $(350 \mathrm{mg} /$ $100 \mathrm{~g}$ ) by intraperitoneal injection for 20 days. On the 10th day, S12 strain group was administered $1.0 \mathrm{ml}$ of S12 $\left(1.5 \times 10^{9} \mathrm{cfu} / \mathrm{ml}\right)$ by gavage for 10 days. On days 0 , 10 and 20 , blood was collected and centrifuged at 4000 $\mathrm{r} / \mathrm{min}$ for 5 mins at $4{ }^{\circ} \mathrm{C}$ to obtain serum. Serum uric acid (UA), urea nitrogen (BUN) and creatinine (Cr) levels were then determined using Hitachi 747 automatic analyzer following standard protocols. At the end of experiments, the rats were sacrificed after being intraperitoneally anesthetized with sodium pentobarbital (65 $\mathrm{mg} / \mathrm{kg}$ ) according to the guidelines for euthanasia in the Guide for Care and Use of Laboratory Animals. The kidney tissues were dissected and fixed in $40 \mathrm{~g} / \mathrm{L}$ paraformaldehyde, wax-embedded and cut into serial sections. The sections were then stained with hematoxylin and eosin (HE), and observed under microscope.

\section{Statistical analysis}

Statistical analysis was performed using SPSS software and GraphPad Prism 5. Data are presented as the mean \pm SD. $P$-value $<0.05$ was considered statistically significant.

\section{Abbreviations}

Cr: Creatinine; HE: Hematoxylin and eosin; LAB: Lactic acid bacteria; UA: Uric acid

\section{Acknowledgements}

We thank School of Pharmacy, Guangzhou Medical University for the use of experimental instrument.

\section{Authors' contributions}

CZ designed the study, YX, XZ and ZY performed the study. All authors have read and approved the manuscript.

\section{Funding}

Fundation: National Natural Science Foundation of China (81973858); Natural Science Foundation of Guangdong Province (2019A1515011200); Science and Technology Project of Qingyuan (2019A028).

Availability of data and materials

All data and material are available upon request to correspondence author

Ethics approval and consent to participate

All animal procedures were performed in accordance with the protocols approved by Animal Care and Use Committee of Guangzhou Medical University (Guangzhou, China).

\section{Consent for publication}

Not applicable.

\section{Competing interests}

The authors declare no conflict of interest.

Received: 8 August 2019 Accepted: 24 June 2020

Published online: 06 July 2020

\section{References}

1. Doghramji PP, Wortmann RL. Hyperuricemia and gout: new concepts in diagnosis and management. Postgrad Med. 2012;124:98-109.

2. Sun SZ, Flickinger BD, Williamson-Hughes PS, Empie MW. Lack of association between dietary fructose and hyperuricemia risk in adults. Nutr Metab (Lond). 2010;7:16.

3. Waigankar SS, Patel V. Role of probiotics in urogenital healthcare. J Midlife Health. 2011;2:5-10.

4. Liu J, Zhang H, Dong Z, Zhou J, Ma Y, Li Y, Qian Q, Yuan Z, Zhang J, Yang Y, Wang $X$, Chen $X$, Zou H, Jin L, Wang J. Mendelian randomization analysis indicates serum urate has a causal effect on renal function in Chinese women. Int Urol Nephrol. 2017:49:2035-42.

5. Borghi $C$. The management of hyperuricemia: back to the pathophysiology of uric acid. Curr Med Res Opin. 2017:33:1-4.

6. Brule D, Sarwar G, Savoie L. Changes in serum and urinary uric acid levels in normal human subjects fed purine-rich foods containing different amounts of adenine and hypoxanthine. J Am Coll Nutr. 2011;3:353-8. 
7. Yun J, Mattsson J, Schnyder K, et al. Allopurinol hypersensitivity is primarily mediated by dose-dependent oxypur-inol-specific T cell response. Clin Exp Allergy. 43(11):1246-55.

8. Collado MC, Meriluoto J, Salminen S. Development of new probiotics by strain combinations: is it possible to improve the adhesion to intestinal mucus. J Dairy Sci. 2007;90:2710-6.

9. Hardin SJ, Singh M, Eyob W, Molnar JC, Homme RP, George AK, Tyagi SC. Diet-induced chronic syndrome, metabolically transformed trimethylamineN-oxide, and the cardiovascular functions. Rev Cardiovasc Med. 2019;20: 121-8.

10. Wang H, Mei L, Deng Y, Liu Y, Wei X, Liu M, Zhou J, Ma H, Zheng P, Yuan J, Li M. Lactobacillus brevis DM9218 ameliorates fructose-induced hyperuricemia through inosine degradation and manipulation of intestinal dysbiosis. Nutrition. 2019;62:63-73.

11. Li M, Yang D, Yuan L, Xie A, Yuan J. Screening and characterization of purine nucleoside degrading lactic acid bacteria isolated from Chinese sauerkraut and evaluation of the serum uric acid lowering effect in hyperuricemia rats. PLoS One. 2014;9:e105577.

12. García-Arroyo FE, Gonzaga G, Muñoz-Jiménez I, et al. Probiotic supplements prevented oxonic acid-induced hyperuricemia and renal damage. PLoS One. 2018;13:e0202901.

13. Yamanaka H, Taniguchi A, Tsuboi H, Kano H, Asami Y. Hypouricaemic effects of yoghurt containing Lactobacillus gasseri PA-3 in patients with hyperuricaemia and/or gout: a randomised, double-blind, placebocontrolled study. Mod Rheumatol. 2019;29:146-50.

14. Tao X, Guan Q Song S, et al. Dynamic changes of lactic acid bacteria flora during Chinese sauerkraut fermentation. Food Control. 2012;26:178-81.

15. Guimaraes AP, Oliveir AA, Ramalho TC. Analysis of bacillus anthracis nucleoside hydrolase via in silico docking with inhibitors and molecular dynamics simulation. J Mol Model. 2011;17:2939-51.

16. González-Franco AC, Robles-Hernández L, Strap JL. Chitinase, chitosanase, and antifungal activities from thermophilic streptomycetes isolated from compost. PHYTON-Int J Exp Botany. 2017:86:14-27.

17. Chou LS, Weimer B. Isolation and characterization of acid-and bile-tolerant isolates from strains of Lactobacillus acidophilus. J Dairy Sci. 1999:82:23-31.

18. Yamada N, Saito-Iwamoto C, Nakamura M, Soeda M, Chiba Y, Kano H, Asami Y. Lactobacillus gasseri PA-3 uses the purines IMP, inosine and hypoxanthine and reduces their absorption in rats. Microoganisms. 2017;5:10.

19. Phuong NT, Lee J, Kang KH, Chang IS, Gadd GM, Kim BH. Analysis of microbial diversity in oligotrophic microbial fuel cells using 165 rDNA sequences. FEMS Microbiol Lett. 2004;233:77-82.

20. Østlie HM, Helland MH, Narvhus JA. Growth and metabolism of selected strains of probiotic bacteria in milk. Int J Food Microbiol. 2003;87:17-25.

21. Iwaki K, Yonetani Y. Hyperuricemic effects of cholinegic agents in rats. Jpn J Pharmacol. 1982;32:343-9.

22. Johnson WJ, Stavric B, Chartrand A. Uricase inhibition in the rat by striazines: an animal model for hyperuricemia and hyperuricosuria. Prodc Soc Exp Biol Med. 1696:131:8-12

23. Berni A, Boddi M, Fattori EB, Cecioni I, Berardino S, Montuschi F, Chiostri M, Poggesi L. Serum uric acid levels and renal damage in hyperuricemic hyperuritensive patients treated with renin-angiotensin system blockers. Am J Hypertens. 2010;23:675-80.

24. Benini A, Marzotto M, Sbarbati A, et al. Assessment of novel probiotic Lactobacillus casei strains for the production of functional dairy foods. Int Dairy J. 2004;14:723-36.

\section{Publisher's Note}

Springer Nature remains neutral with regard to jurisdictional claims in published maps and institutional affiliations.

Ready to submit your research? Choose BMC and benefit from:

- fast, convenient online submission

- thorough peer review by experienced researchers in your field

- rapid publication on acceptance

- support for research data, including large and complex data types

- gold Open Access which fosters wider collaboration and increased citations

- maximum visibility for your research: over $100 \mathrm{M}$ website views per year

At $\mathrm{BMC}$, research is always in progress.

Learn more biomedcentral.com/submissions 